\title{
Obstructive respiratory events and premature atrial contractions after cardioversion
}

\author{
Dominik Linz, Mathias Hohl, Christian Ukena, Felix Mahfoud, Klaus Wirth,
} Hans-Ruprecht Neuberger and Michael Böhm

Affiliation:

Universitätsklinikum des Saarlandes, Klinik für Innere Medizin III, Homburg/Saar, Germany.

\section{Correspondence:}

Dominik Linz, Klinik für Innere Medizin III, Universitätsklinikum des Saarlandes, 66421 Homburg/Saar,

Germany. E-mail: Dominik.LinzQuks.eu

ABSTRACT Recurrence of atrial fibrillation (AF) after electrical cardioversion (ECV) is increased in patients with obstructive sleep apnoea (OSA).

In patients with persistent $\mathrm{AF}$, with $(\mathrm{n}=40)$ and without $(\mathrm{n}=32)$ obstructive respiratory events (OREs) during sedation for ECV, we determined the occurrence of premature atrial contractions (PACs) before and after insertion of a nasopharyngeal tube. The influence of acute obstructive respiratory events on atrial electrophysiology after termination of AF was studied in pigs.

Incidence of PACs directly after ECV was higher in patients with OREs compared to those without OREs ( $7 \pm 2$ versus $1 \pm 1$ per $10 \mathrm{~s}$, respectively; $\mathrm{p}<0.01$ ). Occurrence of PACs could be reduced by $79 \%$ by insertion of a nasopharyngeal tube. In a subsequent sleeping study, patients with OREs had higher apnoea-hypopnoea indices and more PACs during night. 16 patient with and four patients without OREs had a relapse of AF during 1 week after ECV $(\mathrm{p}<0.01)$. In pigs, acute OREs after $30 \mathrm{~min}$ of AF increased occurrence of PACs and vulnerability for reinduction of AF, which could be attenuated by atropine, betablockers and renal denervation.

OREs are associated with increased occurrence of PACs and more early relapse of AF. OREs increase occurrence of PACs and vulnerability for reinduction of AF by sympathovagal imbalance.

$@$ ERSpublications

Obstructive respiratory events aggravate atrial proarrhythmic substrate after termination of atrial fibrillation http://ow.ly/I1xUa

Received: Sept 242014 | Accepted after revision: Dec 102014 | First published online: March 052015

Support statement: D. Linz and M. Hohl are supported by HOMFOR2013/2014. M. Böhm is supported by the Deutsche Forschungsgemeinschaft (KFO 196). D. Linz and F. Mahfoud are supported by the Deutsche Hochdruckliga and Deutsche Gesellschaft für Kardiologie.

Conflict of interest: None declared.

Copyright OERS 2015 


\section{Introduction}

A previous study showed a higher risk of recurrence of atrial fibrillation (AF) after initially successful electrical cardioversion (ECV) in patients with untreated obstructive sleep apnoea (OSA) compared to patients without OSA [1]. Additionally, appropriate treatment of OSA with continuous positive airway pressure was associated with a significant reduction in arrhythmia recurrence [1]. However, the mechanisms by which OSA increases AF recurrence after spontaneous or ECV are unknown. A progressive shortening of atrial refractoriness during the first few days of AF has been shown to increase both the inducibility and persistence of $\mathrm{AF}$ (electrical remodelling) [2, 3]. In a pig model for OSA, negative thoracic pressure during acute obstructive respiratory events (OREs) resulted in a pronounced acute shortening of atrial refractoriness and increased susceptibility to AF in non-remodeled atria [4-6]. Therefore, OREs occurring directly after ECV may result in an additional post-cardioversion shortening of atrial refractoriness, which may enhance the vulnerability for reinduction of AF.

The current study aimed to prospectively evaluate the influence of acute OREs on the occurrence of premature atrial contractions (PACs) and early relapse of AF after ECV in patients with persistent AF. To test whether PACs could induce AF during OREs after termination of AF, the arrhythmogenic electrophysiological substrate for re-entry was investigated in a pig model [4-6]. By treating the animals with the beta-blocker atenolol, the muscarinergic receptor antagonist atropine and modulation of sympathetic nervous system by renal sympathetic denervation [7-9], the role of autonomic nervous system activation was specifically addressed.

\section{Methods}

Electrical cardioversion and ambulatory sleep apnoea screening in patients with atrial fibrillation 72 consecutive patients (patients characteristics see table 1) with symptomatic persistent AF and an Epworth Sleepiness Scale score $>10$ out of 24 were admitted to the hospital between September 2013 and September 2014 and underwent ECV. Two days after ECV, an ambulatory OSA screening was performed in all patients. Patients were scheduled to have their heart rhythm checked 1 week after successful ECV. Patients continued medication after ECV. All patients gave written informed consent and the ECV, which was always clinically indicated in symptomatic persistent AF-patients, was performed following a standard protocol approved by the local institutional committee. The protocol of the human study is presented in figure 1 .

\section{Definition of atrial fibrillation}

Persistent AF was defined as AF documented repeatedly on a 12-lead electrocardiogram (ECG) without intercurrent sinus rhythm at one outpatient visit and directly before ECV.

\section{TABLE 1 Patients characteristics}

\begin{tabular}{lccc} 
Parameters & All & $\begin{array}{c}\text { Obstructive respiratory events during } \\
\text { sedation within 2 min after ECV }\end{array}$ \\
\cline { 2 - 4 } & & $\mathbf{4}$ & - \\
\hline Subjects n & 72 & 40 & 32 \\
Male \% & 54 & 53 & 56 \\
Age years & $55 \pm 3$ & $57 \pm 3$ & $54 \pm 2$ \\
Epworth Sleepiness Scale score & $14 / 24$ & $14 / 24$ & $13 / 24$ \\
Underlying heart disease \% & & & 13 \\
$\quad$ Valvular disease & 15 & 17 & 22 \\
$\quad$ Dilated cardiomyopathy & 23 & 10 & 13 \\
$\quad$ Coronary artery disease & 11 & 50 & 47 \\
$\quad$ Systemic hypertension & 49 & 2 & 2 \\
Arrhythmia history & & 0.9 & 0.7 \\
$\quad$ Median duration months & (range 1-9) & & \\
$\quad$ Previous ECVs per patient $n$ & 0.8 & 80 & 84 \\
Medications during AF \% & & 33 & 38 \\
$\quad$ Beta-blockers & 82 & 80 & 81 \\
$\quad$ Antiarrhythmic drugs & 35 & 33 & 38 \\
Medications after ECV \% & & &
\end{tabular}

There were no significant differences between the groups. ECV: electrical cardioversion; AF: atrial fibrillation; +: events; -: no events. 
Patients with persistent AF

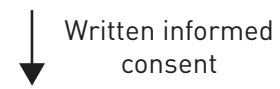

Transoesophageal

echocardiography

Electrical cardioversion

Obstructive respiratory events during deep sedation

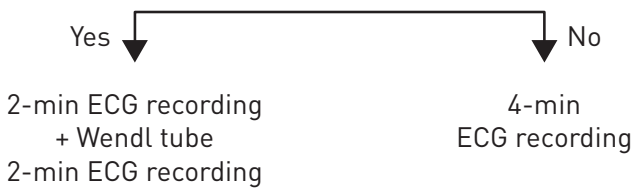

Sleep study

+ Holter ECG

+ Holter ECG

ECG

(Relapse of $A F$ ?)
FIGURE 1 Protocol of the human study. ECG: eletrocardiogram; AF: atrial fibrillation

Electrical cardioversion and definition of ORES

After exclusion of an endocardial thrombus by trans-oesophageal echocardiography, ECV was performed under deep sedation with 10-15 mg midazolam (150 J, biphasic). Blood pressure was measured every 2 min and oxygen saturation was determined continuously. OREs within 2 min after successful ECV were determined visually and were defined as cessation of air flow with maintained breathing efforts for at least $10 \mathrm{~s}$. In all patients with OREs and accompanying oxygen desaturation, a Wendl tube was inserted 2 min after ECV to optimise airway management. OREs were accompanied by a decrease in oxygen saturation of about $15 \%$. After insertion of the Wendl tube, saturation was normalised in $<30 \mathrm{~s}$. Before, during and after ECV, a 12-lead ECG was continuously recorded and the number of PACs recorded. The respective coupling intervals were determined during $4 \mathrm{~min}$ after ECV in 21 patients showing OREs after ECV and in 11 patients not showing OREs after ECV. Flumazenil $(1 \mathrm{mg})$ was applied in all patients at 5 min after ECV. Recurrence of persistent AF within 1 week after ECV was determined by an ambulatory ECG recording. To exclude a time-dependent decrease in the occurrence of PACs during 4 min after ECV, we reanalysed ECG recordings of five additional random patients with OREs, in which a Wendl tube was inserted approximately 4 min after ECV.

\section{Ambulatory OSA screening}

2 days after ECV, an ambulatory sleep study (SomnoScreen plus RC Easy; ResMed, Martinsried, Germany) was performed (recording time $8 \mathrm{~h}$ ) and apnoea-hypopnoea index (AHI) as well as minimal oxygen saturation was determined. ECG recordings were exported and analysed off-line in Microsoft Excel (Microsoft, Redmond, WA, USA). The occurrence of PACs was quantified. The respective coupling intervals were determined in 21 patients showing OREs after ECV and in 11 patients not showing OREs after ECV.

\section{Pig model for atrial fibrillation}

All animal studies were performed in accordance with the Guide for the Care and Use of laboratory Animals published by the US National Institutes of Health (NIH Publication No. 85-23, revised 1996).

In 30 chest-closed male castrated German Landrace pigs (25-30 kg) (anesthetized with 20\% urethane $\left(0.8 \mathrm{~mL} \cdot \mathrm{kg}^{-1}\right.$ intravenous load, $0.4 \mathrm{~mL} \cdot \mathrm{kg}^{-1} \cdot \mathrm{h}^{-1}$ maintenance) and $4 \%$ alpha-chloralose $\left(0.4 \mathrm{~mL} \cdot \mathrm{kg}^{-1}\right.$ i.v. load, $0.1 \mathrm{ml} \cdot \mathrm{kg}^{-1} \cdot \mathrm{h}^{-1}$ maintenance), a tracheotomy was performed to place an endotracheal tube [4]. To deliver atrial tachypacing (basic cycle length $50 \mathrm{~ms}$ ) for $30 \mathrm{~min}$, a 7F bipolar catheter was inserted in the right atrial appendage. 
OSA manoeuvres in pigs

In all animals, negative tracheal pressure (NTP) at -80 mbar was applied by a negative pressure device during tracheal occlusion for 2 min (OSA manoeuvres) to mimic OREs in OSA exactly as described previously [4]. OSA manoeuvres were characterised by intra-apnoeic negative thoracic pressure, hypoxia and hypercapnia and postapnoeic blood pressure rises (see reference [6]). OSA manoeuvres were performed $2 \mathrm{~min}$ after termination of AF induced by $30 \mathrm{~min}$ of atrial tachypacing.

\section{Haemodynamic measurements}

Blood pressure was measured by a TIP catheter (Millar PC 350; Millar Instruments, Houston, TX, USA) in the femoral artery. Bipolar body surface ECG was recorded using subcutaneous needle electrodes in the classical lead II arrangement.

\section{Electrophysiological measurements}

Atrial responses to the pacing procedure were visualised by monophasic action potential (MAP) recordings from the endocardium of the lateral right atrium by a MAP pacing catheter (combined MAP and stimulation catheter, 7 French; Foehr Medical Instruments $\mathrm{GmbH}$, Seeheim, Germany). Atrial effective refractory period (AERP) was measured at a basic cycle length (BCL) of $300 \mathrm{~ms}$ as described previously [4]. During the AERP measurement procedure, the shortest premature S2 extrastimulus resulting in a propagated response frequently induced episodes of AF. When atrial MAP signals showed an irregular rapid activation (cycle length $<200 \mathrm{~ms}$, duration $>5 \mathrm{~s}$ ), AF was diagnosed and used to determine inducible AF duration. During 2 min of tracheal occlusion with applied negative tracheal pressure, the occurrence of PACs and the respective coupling intervals were quantified.

\section{Surgical renal denervation}

In 10 animals, both kidneys were surgically denervated by cutting all visible nerves in the area of the renal hilus and by stripping approximately $1 \mathrm{~cm}$ of the adventitia from the renal artery. The area was then moistened with a $20 \%$ phenol/ethanol solution for 10-15 min. Left renal flow was measured with a Doppler flow probe (transit time flowmeter module system; Transonic Systems Inc., Elsloo, The Netherlands) positioned on the blood vessels. Following surgical renal denervation (RDN), the animals were allowed to re-equilibrate for $1.5 \mathrm{~h}$. Significant reduction of a reproducible postapnoeic blood pressure rise and the absence ( $<5 \%$ change) of a decrease in renal blood flow induced by tracheal occlusion for 2 min were taken as evidence of the completeness of RDN [5, 6]. Sham surgical procedure with kidney exposition without RDN and vehicle for atropine and atenolol (DMSO plus PEG) was performed in 11 other pigs serving as a control.

Drugs

Atenolol $\left(3 \mathrm{mg} \cdot \mathrm{kg}^{-1}, \mathrm{n}=5\right)$ and atropine $\left(0.25 \mathrm{mg} \cdot \mathrm{animal}^{-1}, \mathrm{n}=4\right)$ were dissolved in $0.5 \mathrm{~mL}$ DMSO and $2.5 \mathrm{~mL}$ PEG 400. Solutions were injected over $5 \mathrm{~min}$ before induction of AF. Atenolol was used in a concentration, which resulted in a maximal heart rate reduction in previous studies $[5,6]$.

\section{Statistics}

Data are presented as mean \pm SEM. For comparisons of single repeated measures only, a paired t-test was used. For multiple repeated measures comparisons with the same baseline, repeated-measure two-way ANOVA was used followed by Dunnet's test to compare individual mean difference if ANOVA was significant. A three-way ANOVA with repeated measures was performed to calculate the effect of $30 \mathrm{~min}$ atrial tachypacing on refractoriness, inducible AF duration and PACs, and also to calculate a significant effect of NTP and treatment (atenolol, atropine, RDN), and their interaction using the differences with baseline. If the interaction between NTP and treatment was significant, a two-way ANOVA with repeated measures was performed, followed by Dunnet's test. Fisher's exact test was used to compare relapse of AF. A p-value $<0.05$ was considered significant. For all statistical calculations SAS 8 software (SAS Institute, Cary, NC, USA) was used.

\section{Results}

\section{Clinical observations}

Effect of ORES on cardiac rhythm after electrical cardioversion in patients with persistent AF

Baseline characteristics of the group of 72 consecutive patients ( $55 \pm 3$ years, $54 \%$ male) with an Epworth Sleepiness Scale score $>10$ out of 24 are given in table 1 . When comparing the patients with and without OREs during deep sedation for ECV, patient characteristics did not differ significantly. 
Premature atrial beats and relapse of $A F$

40 of the 72 patients, showed OREs with a desaturation by at least $15 \%$ within 2 min after initially successful ECV during deep sedation (for example, see fig. 2). In all cases, OREs were associated with an increased occurrence of PACs ( $7 \pm 2$ per $10 \mathrm{~s}$, mean coupling interval: $420 \pm 10 \mathrm{~ms}$ ). At 2 min after ECV, a Wendl tube was inserted to prevent OREs and intermittent hypoxia. Within a few seconds, PACs $(2 \pm 0.5$ per $10 \mathrm{~s} ; \mathrm{p}<0.0001)$ were clearly reduced. Occurrence of PACs was low $(1 \pm 1$ per $10 \mathrm{~s})$ in the 32 remaining patients without OREs during deep sedation (fig. 3a). To exclude a spontaneous time-dependent decrease in the occurrence of PACs within the experimental period, a Wendl tube was inserted 4 min after ECV in five other randomly selected patients with OREs. Within the second $2 \mathrm{~min}$, occurrence of PACs was not significantly different compared with the first 2 min after ECV $(6 \pm 3$ per $10 \mathrm{~s}$ versus $8 \pm 3$ per $10 \mathrm{~s}$, respectively; $\mathrm{p}=0.84$ ).

Sleep study

In the selected 40 patients with OREs during deep sedation, OSA severity was higher compared with those patients without OREs during deep sedation (AHI $22 \pm 2$ events $\mathrm{h}^{-1}$ and minimal oxygen saturation $79 \pm 5 \%$ versus AHI $7 \pm 2$ events $\mathrm{h}^{-1}$ and minimal oxygen saturation $91 \pm 3 \%$, respectively; $\mathrm{p}<0.01$ for both) (fig. $3 \mathrm{~b}$ ). Patients with OREs during deep sedation also showed a higher occurrence of PACs during sleep at night (fig. 3b).

\section{Relapse of AF}

16 of the patients with and four of the patients without repetitive OREs during deep sedation immediately after ECV had a relapse of AF during the first week ( $p=0.016$ ) (fig. 4). In the sub-group of patients with OREs during deep sedation and a relapse of AF within 1 week after ECV, mean coupling intervals of PACs occurring during $8 \mathrm{~h}$ of sleep were shorter compared with patients without relapse $(402 \pm 23 \mathrm{~ms}$ versus 478 $\pm 35 \mathrm{~ms}$, respectively; $\mathrm{p}=0.0013$ ).

\section{Experimental studies in pigs}

Effect of OSA manoeuvres on atrial refractoriness after AF termination

During normal breathing, atrial tachypacing for $30 \mathrm{~min}$ shortened AERP by approximately $30 \mathrm{~ms}$ (electrical remodelling). In remodelled atria, OSA manoeuvres resulted in a further immediate transient

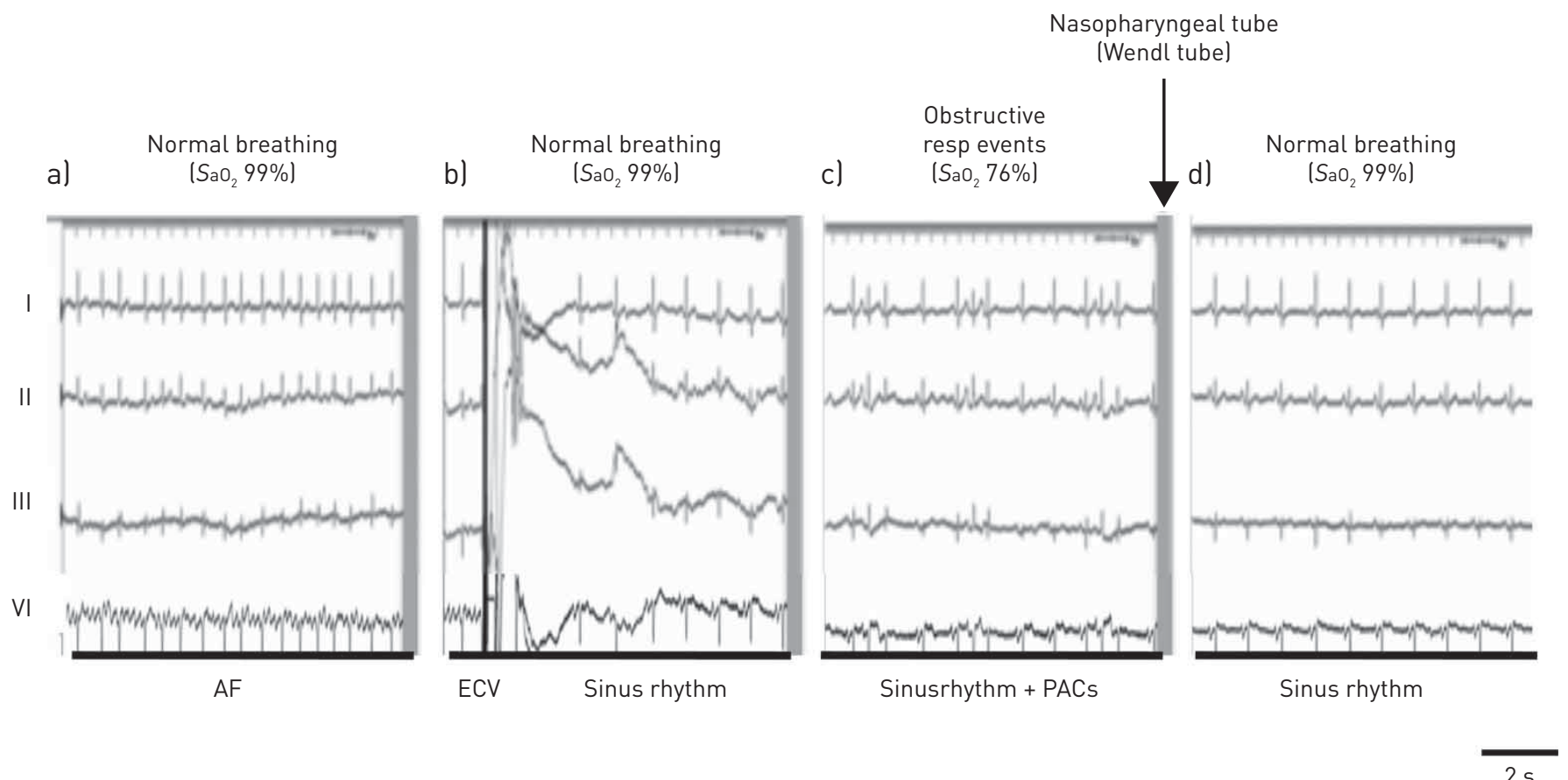

FIGURE 2 Clinical observations. Representative electrocardiogram (ECG)-recordings of an atrial fibrillation (AF) patient with obstructive respiratory events undergoing electrical cardioversion. a) AF during normal breathing and optimal arterial oxygen saturation $\left(\mathrm{SaO}_{2}\right.$ 99\%). b) Electrical cardioversion (ECV) to sinus rhythm during normal breathing $\left(\mathrm{SaO}_{2} 99 \%\right)$. c) Obstructive respiratory events and hypoxia $\left(\mathrm{SaO}_{2} 76 \%\right)$ is associated with increased occurrence of premature atrial contractions (PACs) during sinus rhythm. d) Prevention of obstructive respiratory events and hypoxia by insertion of a nasopharyngeal (Wendl) tube results in stable sinus rhythm again. 


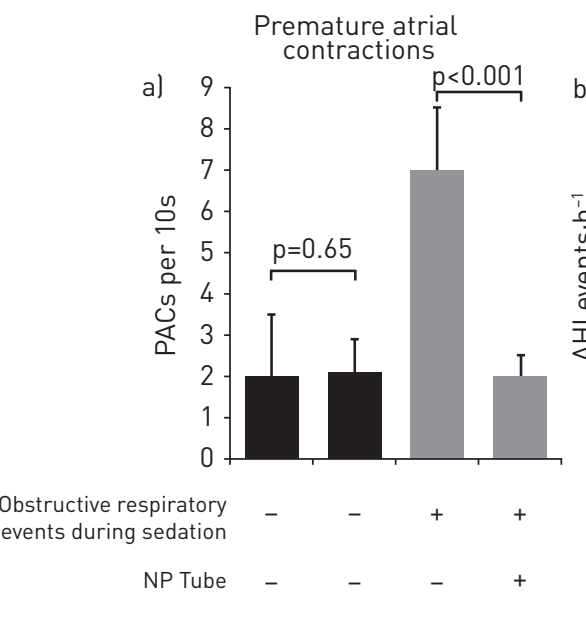

Apnoea-hypopnoea index

PACs
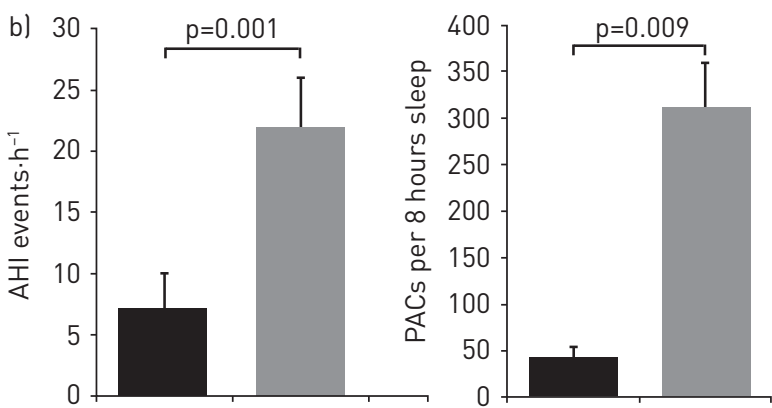

Time after ECV $0-2 \min 3-5 \min 0-2 \min 3-5$ min

FIGURE 3 Clinical observations. a) Premature atrial contractions (PACs) directly after electrical cardioversion (ECV) with or without insertion of a nasopharyngeal (Wendl) tube (NP tube) at 2 min after ECV during sedation and b) apnoea-hypopnoea indices and occurrence of premature atrial contractions (PACs) during sleep 2 days after ECV in patients with (grey) and without (black) obstructive respiratory events during sedation for ECV. Data are presented as mean \pm SEM.

AERP shortening from $117 \pm 12 \mathrm{~ms}$ to $75 \pm 9 \mathrm{~ms}$ ( $\mathrm{p}=0.001$ ) (fig. 5a). Electrical remodelling was not influenced by atropine, atenolol or RDN. AERP shortening induced by OSA manoeuvres in remodelled atria was not modulated by atenolol, partly attenuated by RDN and completely prevented by atropine. There was a different time course of recovery of the AERP shortening induced by OSA manoeuvres ( $\sim 2 \mathrm{~min}$ ) compared with electrical remodelling ( $\sim 20 \mathrm{~min}$ after $30 \mathrm{~min}$ of atrial tachypacing).

\section{Effect of OSA manoeuvres on AF in electrically remodelled atria}

In normal atria, programmed atrial stimulation did not result in AF. In electrically remodelled atria, programmed atrial stimulation induced AF in $80 \%$ during normal breathing and in $100 \%$ during OSA manoeuvres by an atrial premature stimulus with a short coupling interval that did not result in a conducted beat in the baseline state. AF inducibility was not significantly influenced by atenolol, atropine or RDN (data not shown). Application of OSA manoeuvres prolonged inducible AF episodes in remodelled atria. AF episodes were shorter after RDN and after atropine but not significantly modulated by atenolol (fig. 5b).

Effect of OSA manoeuvres on spontaneous PACs after AF termination

During the first $2 \mathrm{~min}$ after spontaneous termination of AF induced by $30 \mathrm{~min}$ of atrial tachypacing (remodelled atrium), a very low number of spontaneous PACs were documented. The application of OSA

FIGURE 4 Clinical observations. Relapse of atrial fibrillation (AF) (black) within 7 days after electrical cardioversion (ECV) in patients with $(+)$ and without $(-)$ obstructive respiratory events during sedation for ECV. SR: sinus rhythm.

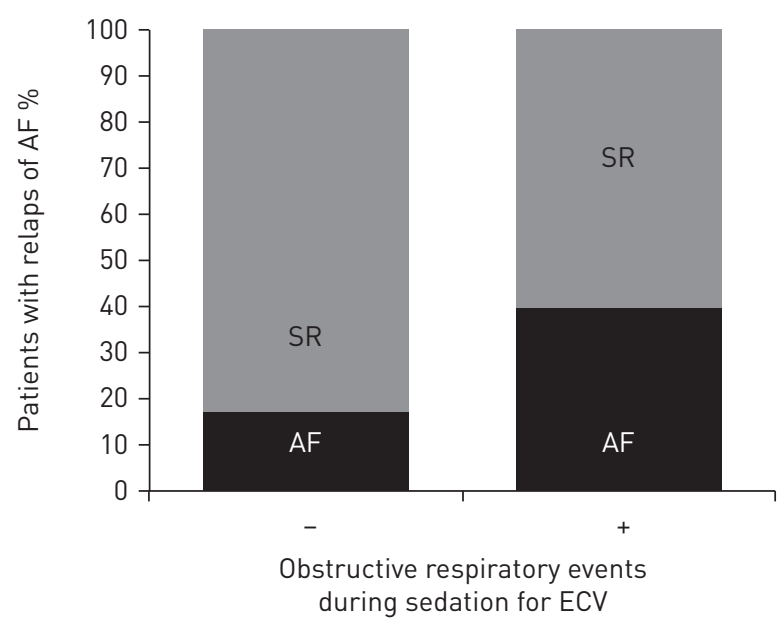


a)
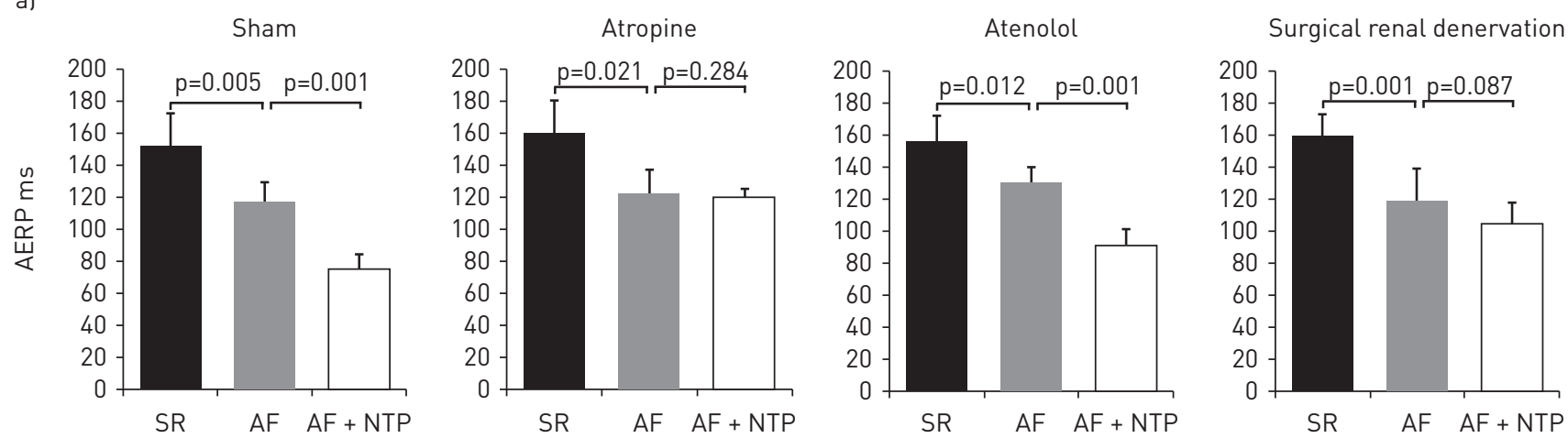

b)
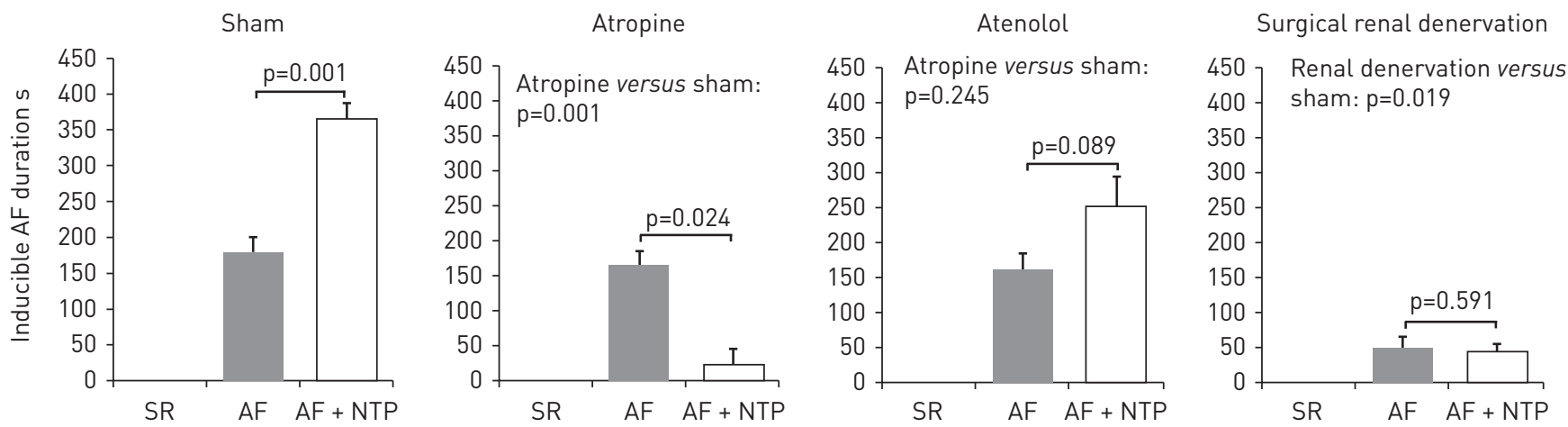

FIGURE 5 Animal study. a) Atrial effective refractory period (AERP) and b) inducible atrial fibrillation (AF) duration in normal atria during sinus rhythm (SR black), in electrical remodelling after $30 \mathrm{~min}$ of atrial tachypacing (AF; grey) and after $2 \mathrm{~min}$ of obstructive respiratory events with applied negative tracheal pressure (AF+NTP; white) in electrical remodelling in pigs with sham operation (sham, $n=11)$, atropine $(n=4)$, atenolol $(n=5)$ and renal denervation $(n=10)$ (basic cycle lengths: $300 \mathrm{~ms}$ ). Data are presented as mean \pm SEM.

manoeuvres significantly increased the number of PACs by $230 \%$. This increase in spontaneous PACs tended to be attenuated by RDN and was not significantly modulated by atropin or atenolol (fig. 6).

\section{Discussion}

Patients with AF have a high prevalence of OSA, and conversely, a high prevalence of AF has been reported in OSA, also in the absence of an underlying cardiac disease [10]. Additionally, a prospective analysis by KANAGALA et al. [11] demonstrated that patients with OSA have a higher recurrence rate of AF after initial successful ECV than patients without OSA. A meta-analysis revealed a significantly higher recurrence of AF following catheter ablation in OSA patients [12]. Patients with severe OSA were less
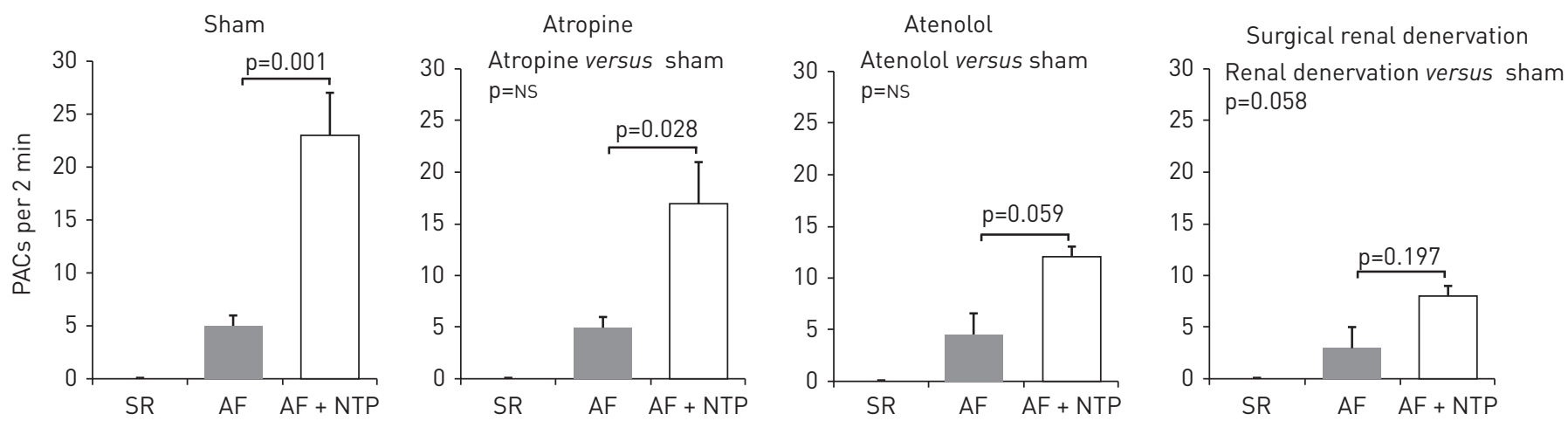

FIGURE 6 Animal study. Occurrence of premature atrial contractions (PACs) in normal atria during sinus rhythm (SR; black), in electrical remodelling after $30 \mathrm{~min}$ of atrial tachypacing (AF; grey) and after $2 \mathrm{~min}$ of obstructive respiratory events with applied negative tracheal pressure (AF+NTP; white) in electrical remodelling in pigs with sham operation (sham, $n=11)$, atropine $(n=4)$, atenolol $(n=5)$ and renal denervation $(n=10)$ (basic cycle lengths: 300 ms). Data are presented as mean \pm SEM. NS: nonsignificant. 
likely to respond to antiarrhythmic therapy for AF than those with milder forms of OSA [13]. Importantly, successful treatment of OSA by continuous positive airway pressure improved catheter ablation success rates [14] and reduced recurrence of AF after ECV in OSA patients [11]. OSA has been shown to be associated with significant atrial remodelling in patients [15]. However, it is unclear whether progressive atrial structural changes alone or with electrophysiological alterations during acute OREs are responsible for AF induction and maintenance in OSA.

In the current study, we showed increased relapse of AF within one week after ECV in patients with OREs. Additionally, we specifically investigated the influence of OREs on the occurrence and characteristics of PACs. Number of PACs within the first 2 min after ECV of persistent AF was higher in patients who showed repetitive OREs during deep sedation compared with those who did not. Prevention of OREs by optimisation of upper airway management by insertion of a nasopharyngeal tube (Wendl tube) reduced occurrence of PACs, suggesting a causal relationship. Interestingly, occurrence of OREs during deep sedation was also associated with higher AHI, more PACs during sleep in a subsequent sleep study and a higher relapse rate of AF within 1 week after ECV suggesting that increased occurrence of PACs may represent a potent trigger for recurrent $\mathrm{AF}$ in OSA [3]. Mapping of immediate recurrence of AF after electrical or spontaneous restoration in patients demonstrated that a majority of $\mathrm{AF}$ episodes were triggered by PACs, mostly originating from atrial foci in the pulmonary veins [16]. Additionally, in ambulatory Holter monitoring, PACs were identified as the general onset mechanism of paroxysmal atrial fibrillation [17]. These findings have also been confirmed in other studies $[18,19]$. In accordance with this observation in humans, spontaneous PAC also represented a sufficient trigger for AF in pig [6] and dog [20] models for sleep apnoea. Additionally, in patients who had a relapse of AF in our study, shorter coupling intervals of PACs were recorded during deep sedation immediately after ECV and during sleep in a subsequent sleeping study.

To mimic PACs with short coupling intervals and to test whether they could induce AF during OREs after termination of AF, we performed programmed atrial stimulation in a pig model for OSA [4-6], which reproducibly resulted in almost $100 \%$ AF inducibility. It is interesting to note that AF was induced by a short-coupled atrial premature stimulus that did not result in a conducted beat before application of OSA manoeuvres. Our observations in the pig model for OSA imply that OREs immediately after termination of AF can create a substrate for the re-initiation of re-entries [3] characterised by a pronounced transient shortening of atrial refractoriness. However, the transient shortening in refractoriness induced by OSA manoeuvres should not be confused with atrial tachypacing-induced electrical remodelling [4]. AF can perpetuate AF by initiation of an electrical remodelling process (atrial electrical remodelling) [2], mainly characterised by calcium-induced inactivation of L-type calcium channels shortening the plateau phase of the action potential [3]. OSA manoeuvres resulted in an additional transient shortening of atrial refractoriness in remodelled atria in pigs. Due to the cumulative effects of atrial tachypacing-induced electrical remodelling and immediate AERP shortening induced by OSA manoeuvres, AERP could become as short as $75 \pm 9 \mathrm{~ms}$. For a premature stimulus to induce AF, heterogeneity of refractoriness is necessary [3]. Immediately after ECV, electrical remodelling and OREs may have shortened the refractory period of the atria to such an extent that particularly PACs with very short coupling intervals result in dispersion of refractoriness creating a substrate for re-entry [3].

Simultaneous sympatho-vagal activation, as observed during OREs during sleep apnoea [21], might be responsible for increased propensity to spontaneous ectopies and AF in OSA $[3,22]$. In the pig model for OSA, spontaneous PACs were inhibited by modulation of autonomic nervous system by RDN. Besides sympatho-vagal activation in OSA, AF itself alters calcium homeostasis, which may favour spontaneous calcium releases $[23,24]$ and play a role for the initiation and perpetuation of AF. Additionally, atropine and RDN prevented the aggravation of the shortening of atrial refractoriness by OREs directly after termination of $\mathrm{AF}$, which was associated with a clear reduction in inducible AF duration. These results support the role of autonomic nervous system for shortening of atrial refractoriness during OREs compared to electrical remodeling during AF induced by atrial tachypacing, which is not modulated by atropine, atenolol or RDN.

\section{Limitations of the study}

OREs within 2 min after ECV were only defined visually but were confirmed afterwards by an ambulatory OSA screening. Nasopharyngeal tubes can splint the upper airway and avoid OREs but the possibility of mucosal stimulation of vagal afferents cannot be excluded. In five random patients with OREs after ECV, occurrence of PACs did not significantly differ between the first and the second 2 min after ECV. This does not completely exclude a spontaneous time-dependent early decrease in the occurrence of PACs in humans but does make it unlikely. We used deep sedation during and after ECV in patients. Central nervous system depression under midazolam sedation is profound. The rate and characteristics of apnoea during less deep sedation is unclear. To overcome this limitation, we performed a sleep study in all 
patients and observed increased occurrence of PACs in patients during sleep even without sedation 2 days after ECV. We used atrial tachypacing to induce atrial electrical remodelling in pigs. Whether our findings in pigs can be completely translated to the clinical setting is unclear.

\section{Conclusion and clinical implications}

Patients who show OREs during deep sedation for ECV also show higher AHIs during sleep and more relapse of AF during the first week after termination of AF. Acute OREs after termination of AF increase occurrence of PACs in patients and in pigs, representing a potential trigger for AF. In atria with atrial tachypacing-induced electrical remodelling, an additional and transient shortening of atrial refractoriness induced by acute OREs creates a highly arrhythmogenic substrate for the initiation and perpetuation of AF in pigs. These OSA-induced electrophysiological changes are mainly driven by sympatho-vagal imbalance, as they could be prevented by atropine and partly attenuated by $\mathrm{RDN}$ in pigs. Effective airway management during and after ECV of AF may reduce early AF recurrence in OSA-patients.

\section{References}

1 Kanagala R, Murali NS, Friedman PA, et al. Obstructive sleep apnea and the recurrence of atrial fibrillation. Circulation 2003; 107: 2589-2594.

2 Wiiffels MC, Kirchhof CJ, Dorland R, et al. Atrial fibrillation begets atrial fibrillation. A study in awake chronically instrumented goats. Circulation 1995; 92: 1954-1968.

3 Schotten U, Verheule S, Kirchhof $\mathrm{P}$, et al. Pathophysiological mechanisms of atrial fibrillation: a translational appraisal. Physiol Rev 2011; 91: 265-325.

4 Linz D, Schotten U, Neuberger HR, et al. Negative tracheal pressure during obstructive respiratory events promotes atrial fibrillation by vagal activation. Heart Rhythm 2011; 8: 1436-1443.

5 Linz D, Mahfoud F, Schotten U, et al. Renal sympathetic denervation suppresses postapneic blood pressure rises and atrial fibrillation in a model for sleep apnea. Hypertension 2012; 60: 172-178.

6 Linz D, Hohl M, Nickel A, et al. Effect of renal denervation on neurohumoral activation triggering atrial fibrillation in obstructive sleep apnea. Hypertension 2013; 62: 767-774.

7 Krum H, Schlaich M, Whitbourn R, et al. Catheter-based renal sympathetic denervation for resistant hypertension: a multicentre safety and proof-of-principle cohort study. Lancet 2009; 373: 1275-1281.

8 Linz D, Ukena C, Mahfoud F, et al. Atrial autonomic innervation: a target for interventional antiarrhythmic therapy? J Am Coll Cardiol 2014; 63: 215-224.

9 Hering D, Lambert EA, Marusic P, et al. Substantial reduction in single sympathetic nerve firing after renal denervation in patients with resistant hypertension. Hypertension 2013; 61: 457-464.

10 Gami AS, Pressman G, Caples SM, et al. Association of atrial fibrillation and obstructive sleep apnea. Circulation 2004; 110: 364-367.

11 Kanagala R, Murali NS, Friedman PA, et al. Obstructive sleep apnea and the recurrence of atrial fibrillation. Circulation 2003; 107: 2589-2594.

12 Ng CY, Liu T, Shehata M, Stevens S, et al. Meta-analysis of obstructive sleep apnea as predictor of atrial fibrillation recurrence after catheter ablation. Am J Cardiol 2011; 108: 47-51.

13 Monahan K, Brewster J, Wang L, et al. Relation of the severity of obstructive sleep apnea in response to anti-arrhythmic drugs in patients with atrial fibrillation or atrial flutter. Am J Cardiol 2012; 110: 369-372.

14 Patel D, Mohanty P, Di Biase L, et al. Safety and efficacy of pulmonary vein antral isolation in patients with obstructive sleep apnea: the impact of continuous positive airway pressure. Circ Arrhythm Electrophysiol 2010; 3: $445-451$.

15 Dimitri $\mathrm{H}, \mathrm{Ng} \mathrm{M}$, Brooks $\mathrm{AG}$, et al. Atrial remodeling in obstructive sleep apnea: implications for atrial fibrillation. Heart Rhythm 2012; 9: 321-327.

16 Haïssaguerre M, Jais P, Shah DC, et al. Catheter ablation of chronic atrial fibrillation targeting the reinitiating triggers. J Cardiovasc Electrophysiol 2000; 11: 2-10.

17 Vincenti A, Brambilla R, Fumagalli MG, et al. Onset mechanism of paroxysmal atrial fibrillation detected by ambulatory Holter monitoring. Europace 2006; 8: 204-210.

18 Bennett MA, Pentecost, BL. The pattern of onset and spontaneous cessation of atrial fibrillation in man. Circulation 1970; 41: 981-988.

19 Hnatkova K, Waktare JE, Murgatroyd FD, et al. Analysis of the cardiac rhythm preceding episodes of paroxysmal atrial fibrillation. Am Heart J 1998; 135: 1010-1019.

20 Ghias M, Scherlag BJ, Lu Z, et al. The role of ganglionated plexi in apnea-related atrial fibrillation. J Am Coll Cardiol 2009; 54: 2075-2083.

21 Kasai T, Bradley TD. Obstructive sleep apnea and heart failure: pathophysiologic and therapeutic implications. J Am Coll Cardiol 2011; 57: 119-127.

22 Sharifov OF, Fedorov VV, Beloshapko GG, et al. Roles of adrenergic and cholinergic stimulation in spontaneous atrial fibrillation in dogs. J Am Coll Cardiol 2004; 43: 483-490.

23 Patterson E, Lazzara R, Szabo B, et al. Sodium-calcium exchange initiated by the Ca2+ transient: an arrhythmia trigger within pulmonary veins. J Am Coll Cardiol 2006; 47: 1196-1206.

24 Vest JA, Wehrens XH, Reiken SR, et al. Defective cardiac ryanodine receptor regulation during atrial fibrillation. Circulation 2005; 111: 2025-2032. 